\title{
Growth of gold flowers on polyacrylonitrile fibers
}

\author{
Zhi-Chuan Wu • Bo Wang • Yong Zhang • \\ Jin-Juan Wang • Ting-Xian Tao
}

Received: 9 November 2008/Accepted: 15 November 2008

(C) Springer Science+Business Media B.V. 2009

This article is retracted because it was published online erroneously in 2008. The Editor-in-Chief did not accept this paper for publication.

This article is retracted because it was published online erroneously in 2008. The Editor-in-Chief did not accept this paper for publication.

Z.-C. Wu ( ()$\cdot$ B. Wang · Y. Zhang · J.-J. Wang

T.-X. Tao

Department of Bioengineering \& Chemical Engineering,

Anhui University of Technology and Science,

Wuhu 241000, People's Republic of China

e-mail: wuzhichuan@auts.edu.cn 